Article

\title{
Lung-Targeted Delivery of Dimethyl Fumarate Promotes the Reversal of Age-Dependent Established Lung Fibrosis
}

\author{
Kosuke Kato ${ }^{1}$ (D), Ioannis Papageorgiou ${ }^{1}$, Yoon-Joo Shin ${ }^{1}$, Jennifer M. Kleinhenz ${ }^{1}$, Sunny Palumbo ${ }^{2}$, \\ Seongmin Hahn ${ }^{2}$, Joseph D. Irish ${ }^{2}$, Skye P. Rounseville ${ }^{2}$, Kenneth S. Knox ${ }^{3}$ and Louise Hecker ${ }^{1,4, *}$ \\ 1 Division of Pulmonary, Allergy and Critical Care and Sleep Medicine, Department of Medicine, \\ Emory University, Atlanta, GA 30322, USA; kosuke.kato@emory.edu (K.K.); \\ ioannis.papageorgiou@emory.edu (I.P.); yoon.joo.shin@emory.edu (Y.-J.S.); \\ jennifer.m.kleinhenz@emory.edu (J.M.K.) \\ 2 Division of Pulmonary, Allergy and Critical Care and Sleep Medicine, Department of Medicine, \\ University of Arizona, Tucson, AZ 85721, USA; sunl@arizona.edu (S.P.); shahn@arizona.edu (S.H.); \\ jirish12@arizona.edu (J.D.I.); srounseville@arizona.edu (S.P.R.) \\ 3 Division of Pulmonary, Allergy and Critical Care and Sleep Medicine, Department of Medicine, \\ University of Arizona College of Medicine-Phoenix, Phoenix, AZ 85004, USA; \\ kknox@deptofmed.arizona.edu \\ 4 Atlanta VA Healthcare System, Atlanta, GA 30033, USA \\ * Correspondence: louise.hecker@emory.edu; Tel.: +1-(404)-727-3689
}

\section{check for} updates

Citation: Kato, K.; Papageorgiou, I.; Shin, Y.-J.; Kleinhenz, J.M.; Palumbo, S.; Hahn, S.; Irish, J.D.; Rounseville, S.P.; Knox, K.S.; Hecker, L. Lung-Targeted Delivery of Dimethyl Fumarate Promotes the Reversal of Age-Dependent Established Lung Fibrosis. Antioxidants 2022, 11, 492. https://doi.org/10.3390/ antiox11030492

Academic Editor: Tsuyoshi Shuto

Received: 20 January 2022

Accepted: 24 February 2022

Published: 28 February 2022

Publisher's Note: MDPI stays neutral with regard to jurisdictional claims in published maps and institutional affiliations.

Copyright: (C) 2022 by the authors. Licensee MDPI, Basel, Switzerland. This article is an open access article distributed under the terms and conditions of the Creative Commons Attribution (CC BY) license (https:// creativecommons.org/licenses/by/ $4.0 /)$.

\begin{abstract}
Idiopathic pulmonary fibrosis (IPF), a severe and deadly form of lung fibrosis, is widely regarded as a disease of aging. We previously demonstrated that aged mice with persistent lung fibrosis and IPF lung myofibroblasts exhibit deficient Nrf2-mediated antioxidant responses. Tecfidera is an orally administered FDA-approved drug for the treatment of multiple sclerosis, where the active pharmaceutical ingredient is dimethyl fumarate (DMF), an active Nrf2 activator. However, no studies have evaluated the efficacy of DMF for age-associated persistent lung fibrosis. Here, we demonstrate that in IPF lung fibroblasts, DMF treatment inhibited both TGF- $\beta$-mediated pro-fibrotic phenotypes and led to a reversal of established pro-fibrotic phenotypes. We also evaluated the pre-clinical efficacy of lung-targeted (inhaled) vs. systemic (oral) delivery of DMF in an aging murine model of bleomycin-induced persistent lung fibrosis. DMF or vehicle was administered daily to aged mice by oral gavage or intranasal delivery from 3-6 weeks post-injury when mice exhibited non-resolving lung fibrosis. In contrast to systemic (oral) delivery, only lung-targeted (inhaled) delivery of DMF restored lung Nrf2 expression levels, reduced lung oxidative stress, and promoted the resolution of age-dependent established fibrosis. This is the first study to demonstrate the efficacy of lung-targeted DMF delivery to promote the resolution of age-dependent established lung fibrosis.
\end{abstract}

Keywords: idiopathic pulmonary fibrosis; dimethyl fumarate; Nrf2; aging; antioxidant; reactive oxygen species

\section{Introduction}

Human fibrotic disorders affect many organ systems, including the heart, blood vessels, kidneys, liver, and lungs [1,2]. An estimated $45 \%$ of deaths in the U.S. are attributable to disorders that are characterized by varying degrees of fibrosis [2]. The most severe form of lung fibrosis is idiopathic pulmonary fibrosis (IPF), a fatal and relentlessly progressive disorder [3,4]. IPF is characterized by excessive scar tissue formation and irreversible destruction of the lung parenchyma, resulting in gas-exchange abnormalities and ultimately respiratory failure. Although two drugs have gained FDA approval for IPF (nintedanib and pirfenidone), neither are shown to definitively improve quality of life or survival. Further, although both drugs are shown to inhibit the development of fibrosis, we recently reported that nintedanib failed to demonstrate efficacy for reversing age-associated established lung 
fibrosis [5]. Clearly, improved therapies for the treatment of IPF and other fibrotic diseases are needed.

As the average life expectancy continues to increase, the elderly population is growing at a rapid pace. Progressive fibrosis is a hallmark of aging in various organ systems, including the liver [6], kidneys [7], pancreas [8], and lungs [9]. With this shift in the elderly demographic, it has become increasingly important to understand the contribution of aging to disease pathogenesis. IPF is widely regarded as a disease of aging [10-12]. The incidence and prevalence of IPF increase with age; two-thirds of IPF patients are older than 60 years at the time of presentation, with a mean age of 66 years at the time of diagnosis [4] Further, the survival rate for IPF patients markedly decreases with age [10]. Despite this strong association, cellular/molecular mechanisms that account for the aging predilection to fibrotic disease are not well understood.

Key mechanisms of pathological fibrosis appear to be common among all tissues/organs, in particular oxidative stress and myofibroblast activation. Oxidative stress is defined as an imbalance between reactive oxygen species (ROS) production and antioxidant capacity. Numerous studies implicated defective antioxidant responses in the pathogenesis of IPF [13-18]. The induction of the nuclear factor erythroid 2-related factor 2 (Nrf2) serves as a master regulator of the cellular antioxidant responses by inducing the transcription of a wide array of genes that can mitigate oxidative stress. However, patients with IPF exhibit decreased Nrf2 expression and redox imbalance that promotes pro-fibrogenic responses in myofibroblasts [19-21]. In animal models of pulmonary fibrosis, Nrf2 deficiency results in heightened oxidative stress and more severe pulmonary fibrosis [22-24]. Our previous studies demonstrated that aged mice exhibited an impaired capacity for fibrosis resolution; this is in part regulated by alterations in cellular redox homeostasis resulting from deficient induction of Nrf2 in lung fibroblasts [19]. Although the efficacy of antioxidant-targeted strategies via Nrf2 activation is shown to suppress the development of bleomycin-induced lung fibrosis in young animals $[25,26]$, the efficacy of Nrf2 activation for reversing agedependent established lung fibrosis has never been evaluated.

Although therapeutic strategies targeting Nrf2 for the treatment of IPF hold promise, systemic delivery of antioxidant-targeted strategies (such as N-Acetylcysteine) failed in clinical trials for IPF (NCT00639496) [27]. Further, systemic targeting of Nrf2 has a high potential for off-target effects as well as safety concerns; systemic delivery of the Nrf2 activator, bardoxolone methyl, was terminated in phase III clinical trials due to safety concerns (NCT01351675). Tecfidera (developed by Biogen) is an FDA-approved drug for the treatment of multiple sclerosis via the oral route of administration; the active pharmaceutical ingredient in Tecfidera is dimethyl fumarate (DMF), an Nrf2-activator [28]. There is a critical need for antioxidant-based therapies that can promote the resolution of established lung fibrosis without deleterious adverse effects. The goal of this study was to determine the pre-clinical efficacy of lung-targeted delivery vs. systemic delivery of DMF in an aging murine model of persistent lung fibrosis.

\section{Materials and Methods}

\subsection{Reagents}

We purchased the following reagents: Dimethyl sulfoxide (DMSO) from Mediatech (Tewksbury, MA, USA); Hank's Balanced Salt Solution (HBSS, -Ca/Mg), Dulbecco's Modified Eagle Medium (DMEM), and DMF from Fisher Scientific (Waltham, MA, USA); Penicillin/streptomycin solution and fetal bovine serum (FBS) from Gibco (Grand Island, NY, USA); Amplex Red reagent from Molecular Probes (Eugene, Oregon); CellTiter-Glo ${ }^{\circledR}$ Luminescent reagent from Promega (Madison, WI, USA); human recombinant TGF- $\beta 1$ from R\&D Systems (Minneapolis, MN, USA). We purchased the following antibodies: anticollagen- $1 \alpha$ from Invitrogen (Carlsbad, CA, USA); anti-fibronectin-1, anti-Nrf2, and anti$\beta$-actin from Abcam (Cambridge, MA, USA); anti-GAPDH from Cell Signaling (Danvers, MA, USA); horseradish peroxide-conjugate secondary antibodies from Bio-Rad (Hercules, 
CA, USA). All other chemicals and reagents were purchased from Sigma-Aldrich unless otherwise specified.

\subsection{Cell Culture}

Fibroblasts were isolated from the lung of a patient with IPF using collagenase digestion as previously described [19] under protocols approved by the institutional review boards of the University of Arizona (IRB protocol \#1200000347). All cells were cultured in DMEM supplemented with $10 \% \mathrm{FBS}, 100 \mathrm{U} / \mathrm{mL}$ penicillin, $100 \mu \mathrm{g} / \mathrm{mL}$ streptomycin, and $1.25 \mu \mathrm{g} / \mathrm{mL}$ amphotericin $\mathrm{B}$, at $37{ }^{\circ} \mathrm{C}$ in $5 \% \mathrm{CO}_{2}, 95 \%$ air [19].

\subsection{Amplex Red Assay}

Hydrogen peroxide $\left(\mathrm{H}_{2} \mathrm{O}_{2}\right)$ levels in blood and $\mathrm{H}_{2} \mathrm{O}_{2}$ generated by fibroblasts were determined by the Amplex Red assay kit (Life Technologies, Eugene, OR) as previously described [29]. The fluorescence intensity was measured at $525 \mathrm{~nm}$ for excitation and emission in the range of 580-640 $\mathrm{nm}$ using the Glomax multi detection system (Promega, Madison, WI, USA).

\subsection{Western Blotting}

We prepared cell lysates and lung homogenates in RIPA buffer, subjected them to SDS-PAGE under reducing conditions, and performed Western blotting as previously described [30]. Lysates were quantitated using a Micro BCA Protein assay kit (Pierce, Waltham, MA, USA) according to the manufacturer's instructions. We used enhanced chemiluminescence Western blotting substrate and c400 Imager (Azure Biosystems, Dublin, CA, USA) to detect specific immunoreactive signals. Densitometric analysis was performed using the NIH ImageJ software.

\subsection{Murine Model of Bleomycin-Induced Lung Injury}

Aged (18 months) female C57BL/6J mice (The Jackson Laboratory) were anesthetized using intraperitoneal injection of ketamine $(100 \mathrm{mg} / \mathrm{kg})$ and xylazine $(10 \mathrm{mg} / \mathrm{kg})$. Bleomycin $(0.02875 \mathrm{U} /$ mouse; $50 \mu \mathrm{L})$ was administered intratracheally to induce lung fibrosis as previously described [19]. Since gender differences exist in response to injury in this model [31], we utilized female mice only to avoid sex-related variability of responses to injury.

\subsection{Therapeutic Treatments}

At 3 weeks post-bleomycin administration, mice were randomly assigned to one of four treatment groups. Between 3-6 weeks post-injury, DMF or vehicle (sterile PBS) was administered daily by oral gavage ( $240 \mu \mathrm{g} /$ dose in $150 \mu \mathrm{L}$ total volume) (Table 1$)$ using a gavage tube. In parallel, mice were anesthetized using isoflurane and administered DMF or vehicle (sterile PBS) daily by intranasal instillation $(80 \mu \mathrm{g} /$ dose in $50 \mu \mathrm{L}$ total volume) (Table 1). The control animals received vehicle only. Dosing was based on the solubility of this compound in the vehicle in the total volume used for administration. All mice were monitored for body-weight changes and survival. Mice were removed from the study when we observed greater than $20 \%$ weight loss compared to pre-surgery (day 0 ) or lack of responsiveness to touch. At 6 weeks post-injury, all mice were sacrificed by $\mathrm{CO}_{2}$ inhalation. All procedures were approved by the Institutional Animal Care and Use Committee (IACUC, \#14-535 approved on 17 July 2017) at the University of Arizona.

Table 1. DMF Formulation Protocol.

\begin{tabular}{cccc}
\hline Delivery Route & Vehicle & Volume & Dose \\
\hline Intranasal & PBS & $50 \mu \mathrm{L}$ & $80 \mu \mathrm{g}$ \\
Oral gavage & PBS & $150 \mu \mathrm{L}$ & $240 \mu \mathrm{g}$ \\
\hline
\end{tabular}

DMF was dissolved in sterile PBS at $42{ }^{\circ} \mathrm{C}$ and sonicated for $50 \mathrm{~min}$. 


\subsection{Oxidized Glutathione Assay}

Oxidized glutathione levels in the lung homogenates were determined by the glutathione assay kit (Cayman Chemicals, Ann Arbor, MI, USA) according to the manufacturer's instructions. Oxidized glutathione was normalized to the total protein content in the lung homogenate.

\subsection{Histology}

The lungs were fixed overnight in $10 \%$ neutral buffered formalin, processed, embedded in paraffin, and the sections were stained with hematoxylin and eosin (H\&E) or Masson's trichrome staining was performed [30].

\subsection{Hydroxyproline Assay}

The lungs were weighed, homogenized in sterile water, and hydrolyzed in $12 \mathrm{~N}$ $\mathrm{HCl}$ at $120^{\circ} \mathrm{C}$ for $3 \mathrm{~h}$. A hydroxyproline assay (Sigma-Aldrich, St. Louis, MO, USA) was performed according to the manufacturer's instructions using hydroxyproline as a standard. Hydroxyproline concentration was normalized to the total protein content in the lung homogenate.

\subsection{Lipid Peroxidation Assay}

Oxidized lipid levels in the lung homogenates were determined by the Lipid peroxidation assay kit (Cayman Chemicals, Ann Arbor, MI, USA) according to the manufacturer's instructions. Oxidized lipid concentration was normalized to the total protein content in the lung homogenate.

\subsection{Statistical Analysis}

Graphs were generated, and statistical analyses were performed with GraphPad Prism (GraphPad Software, San Diego, CA, USA). Data are expressed as means \pm SEM. Differences among groups were assessed with one-way ANOVA multiple comparisons with a Tukey's post-test and between pairs with Student's two-tailed $t$-test. $p<0.05$ is considered statistically significant $\left({ }^{*} p<0.05,{ }^{* *} p<0.01\right.$, and $\left.{ }^{* * *} p<0.001\right)$.

\section{Results}

\subsection{DMF Treatment Reduces ROS Levels in IPF Lung Fibroblasts}

Numerous studies implicated defective antioxidant responses in the pathogenesis of IPF [13-18]. In IPF lung fibroblasts, decreased Nrf2 expression was associated with profibrogenic phenotypes [20]. Further, Nrf2 activation (via sulforaphane treatment) restored antioxidant responses and promoted dedifferentiation of IPF myofibroblast [20]. In line with these findings, our previous studies demonstrated that IPF lung myofibroblasts exhibit defective Nrf2 responses associated with oxidative stress [19]. DMF treatment was shown to potently diminish pro-fibrotic phenotypes in lung fibroblasts isolated from patients with systemic sclerosis [25]. However, the efficacy of DMF was not previously evaluated in IPF lung fibroblasts. Since primary mesenchymal cells isolated from IPF lungs represent a heterogeneous population of both fibroblasts and myofibroblasts "(myo)fibroblasts", we sought to evaluate the efficacy of DMF on both TGF- $\beta$-induced and established profibrogenic phenotypes of these cells. To determine if DMF treatment can inhibit fibrogenic responses induced by TGF- $\beta$, cells were pre-treated with DMF or vehicle followed by treatment with TGF- $\beta$ for $24 \mathrm{~h}$. DMF treatment significantly reduced TGF- $\beta$-induced production of ROS, specifically $\mathrm{H}_{2} \mathrm{O}_{2}$, as compared to vehicle treatment (Figure 1A). DMF treatment also led to the inhibition of TGF- $\beta$-induced expression of collagen- $1 \alpha$, a major ECM component in IPF fibroblasts (Figure 1B, Supplemental Figure S1). Since primary IPF lung (myo)fibroblasts exhibit enhanced production of ROS and ECM [32], we next sought to determine if DMF can reverse these established pro-fibrotic phenotypes. DMF treatment significantly inhibited the production of $\mathrm{H}_{2} \mathrm{O}_{2}$ (Figure 1C) and collagen-1 $\alpha$ (Figure 1D, Supplemental Figure S1), as compared to vehicle treatment. These results support the 
concept that activation of Nfr2 by DMF could be a therapeutic approach to target redox imbalance and fibrogenic responses in IPF.

A

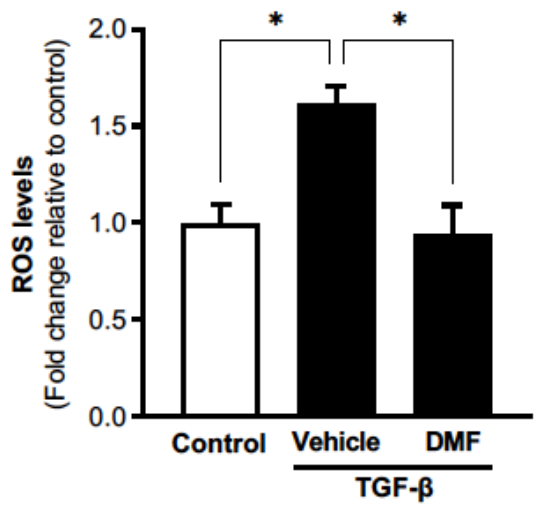

C

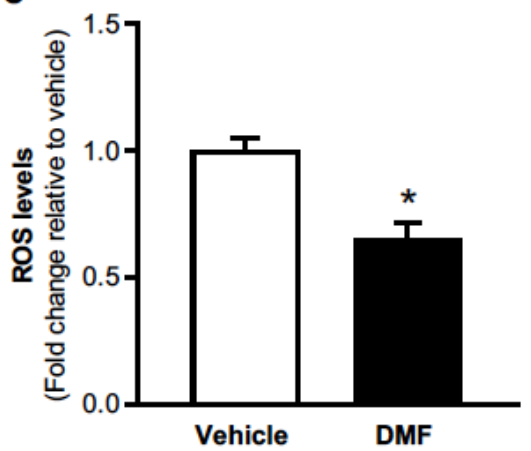

B

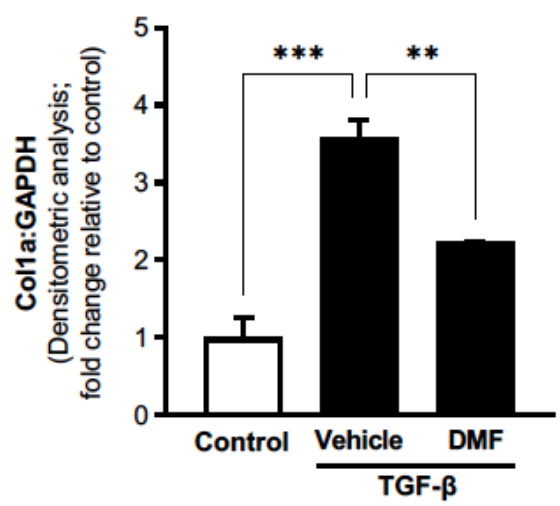

D

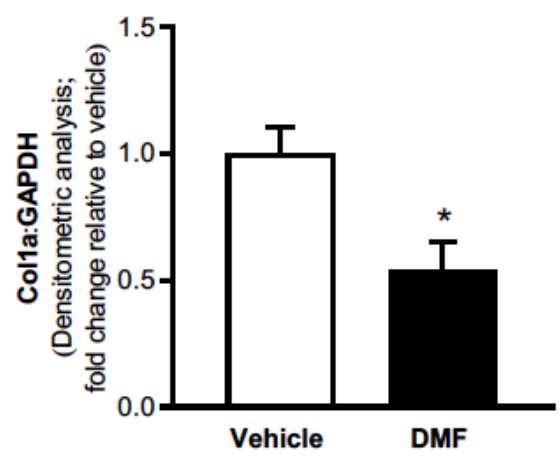

Figure 1. DMF treatment inhibits pro-fibrotic phenotypes in IPF lung fibroblasts. Primary fibroblasts were isolated from the lung of a patient with biopsy-proven IPF. (A,B) IPF lung fibroblasts were treated with DMF $(1 \mu \mathrm{M})$ or vehicle (DMSO) followed by treatment \pm TGF- $\beta(2 \mathrm{ng} / \mathrm{mL}) . \mathrm{H}_{2} \mathrm{O}_{2}$ levels were evaluated at $24 \mathrm{~h}$ by Amplex Red assay (A), and whole-cell lysates were assessed for collagen- $1 \alpha$ expression at $48 \mathrm{~h}$ by Western blot; densitometric analyses are shown (B). (C,D) IPF lung fibroblasts were treated with DMF $(1 \mu \mathrm{M})$ or vehicle (DMSO). Culture supernatant was evaluated for $\mathrm{H}_{2} \mathrm{O}_{2}$ levels at $24 \mathrm{~h}$ by Amplex Red assay (C), and whole-cell lysates were assessed for collagen- $1 \alpha$ expression at $24 \mathrm{~h}$ by Western blot; densitometric analyses are shown (D). All values represent means \pm SEM; technical replicates $(n=3) ;{ }^{*} p<0.05 ;{ }^{* *} p<0.01 ;{ }^{* * *} p<0.01$ using Student's two-tailed $t$-test to compare the means between two groups and one-way ANOVA multiple comparisons with a Tukey's post-test to compare the means among three groups.

\subsection{Oral DMF Delivery Fails to Reverse Age-Dependent Persistent Lung Fibrosis}

In young mice, bleomycin-induced injury results in a self-limited fibrotic response, where fibrosis spontaneously resolves following peak injury $[19,33]$. Conversely, aged mice exhibit a persistent fibrotic response, with little to no resolution of fibrosis from 3 weeks to 4 months post-injury [19]. We previously demonstrated that Nrf2 levels are highly upregulated in young mice following bleomycin-induced injury, which promotes fibrosis resolution [19]. In contrast, the lack of fibrosis resolution in aged mice is associated with a deficient Nrf2 response, where Nrf2 levels are significantly downregulated [19]. Although a recent study demonstrated that daily intraperitoneal administration of DMF inhibited the development of bleomycin-induced lung fibrosis in young mice [25], no studies evaluated whether DMF can reverse age-dependent established lung fibrosis (which is associated with deficient Nrf2 levels). We first sought to confirm that aged mice exhibit a deficient Nrf2 response following bleomycin-induced lung injury. As expected, aged mice demonstrated a significant decrease in lung Nrf2 expression levels at 3 weeks post-injury (Figure 2A,B), which was accompanied by increased levels of oxidized glutathione (Figure 2C), and 
increased expression of ECM markers, including fibronectin (Figure 2D,E) and collagen- $1 \alpha$ (Figure 2F,G). This aging model of bleomycin-induced persistent lung fibrosis enables the evaluation of therapeutic agents for their efficacy in reversing age-dependent established fibrosis [5]. Therefore, we sought to determine whether systemic delivery of DMF (via oral gavage) demonstrates efficacy for reversing age-dependent established fibrosis. Starting at 3 weeks post-injury (when aged mice exhibit established/persistent fibrosis) [19], DMF or vehicle was administered by oral gavage daily through 6 weeks (Figure 3). Similar to our previously reported findings [19], aged vehicle-treated mice demonstrated persistent lung fibrosis at 6 weeks post-injury, as demonstrated by histopathology (Figure 4A; top panels), lung collagen deposition (Figure 4A; bottom panels), elevated fibronectin (Figure 4B,C) and collagen- $1 \alpha$ (Figure $4 \mathrm{D}, \mathrm{E}$ ) expression levels, and elevated lung hydroxyproline levels (Figure 4 F). However, oral DMF treatment failed to promote the resolution of age-dependent established lung fibrosis during this 3-week treatment period, as lung fibrosis in mice treated with oral DMF was indistinguishable from vehicle-treated mice (Figure 4A-F). Overall, systemic (oral) administration of DMF failed to reverse age-dependent established lung fibrosis.

A
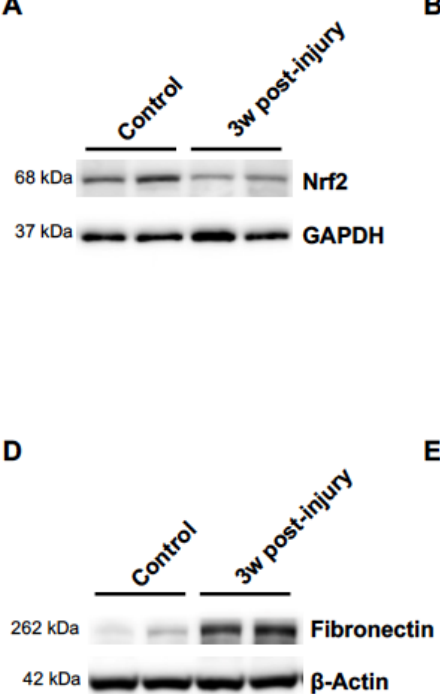

$\mathbf{F}$

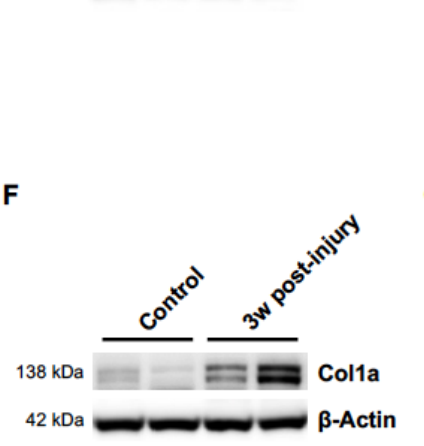

B
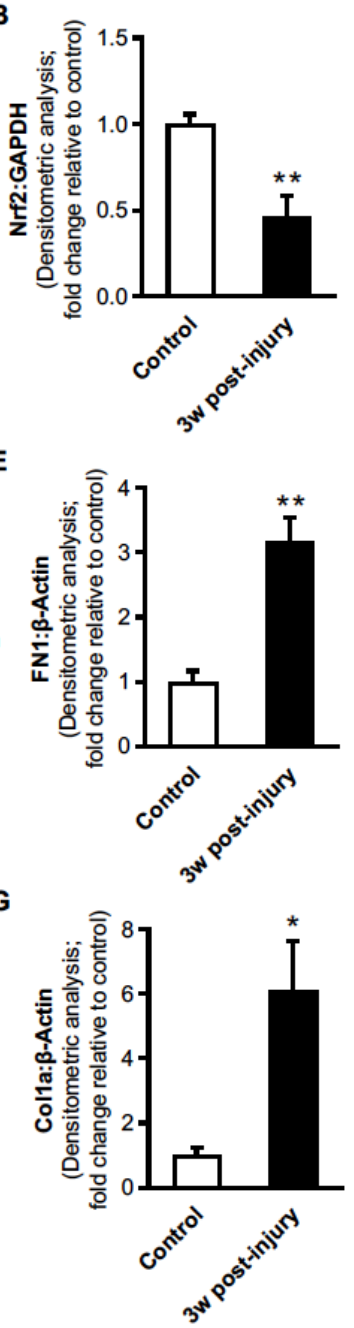

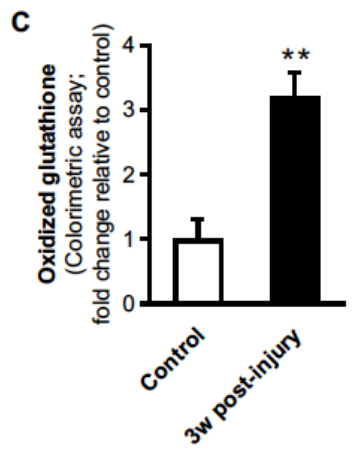

Figure 2. Aged-injured mice exhibit Nrf2 deficiency associated with oxidative stress and lung fibrosis. (A,B) Lung protein was assessed for Nrf2 expression by Western blot (A) and densitometric analysis (B). (C) The level of lung oxidized glutathione was analyzed by quantitative oxidized glutathione assay. (D-G) Lung protein was assessed for fibronectin (D,E) and collagen- $1 \alpha(\mathrm{Col}-1 \alpha)$ $(\mathbf{F}, \mathrm{G})$ expression by Western blot and densitometric analysis. Representative Western blot images are shown. All values represent means of biological replicates \pm SEM; $n=4$ mice per group; ${ }^{*} p<0.05$; ** $p<0.01$ using Student's two-tailed $t$-test. 


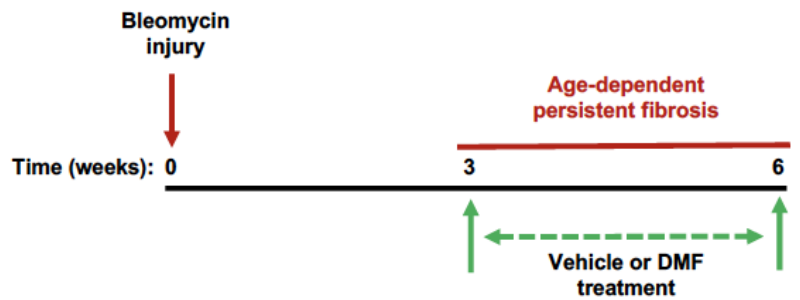

Figure 3. Schematic diagram illustrating age-dependent persistent lung fibrosis and treatment protocol. C57BL/6J aged (18 months) female mice received an intratracheal instillation of bleomycin (0.02875 U/mouse). DMF was administered daily by oral gavage $(240 \mu \mathrm{g} / 150 \mu \mathrm{L}$ in sterile PBS/mouse) or intranasal instillation $(80 \mu \mathrm{g} / 50 \mu \mathrm{L}$ in sterile PBS/mouse) from 3-6 weeks post-injury.

A

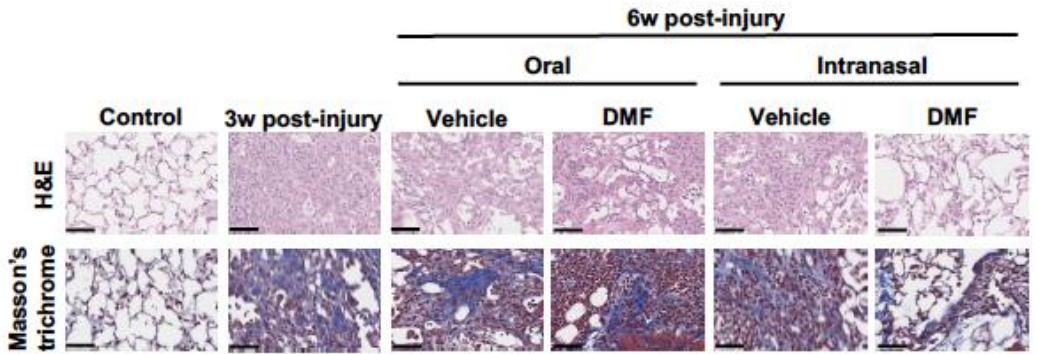

B

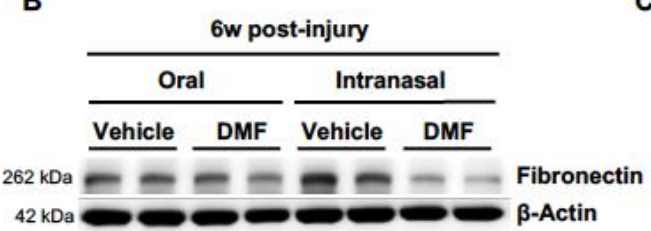

D

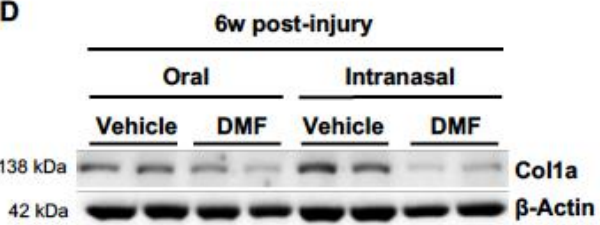

F

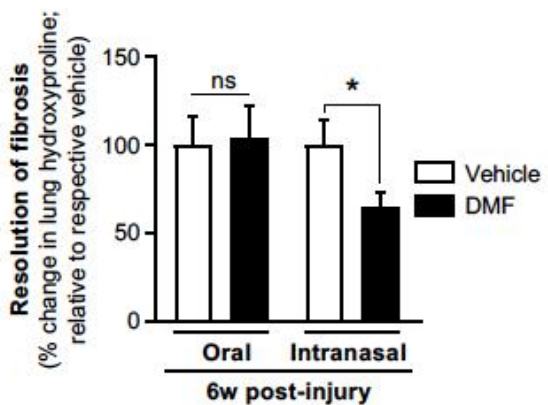

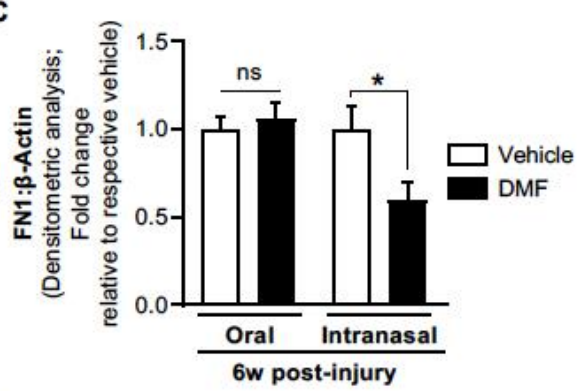

E

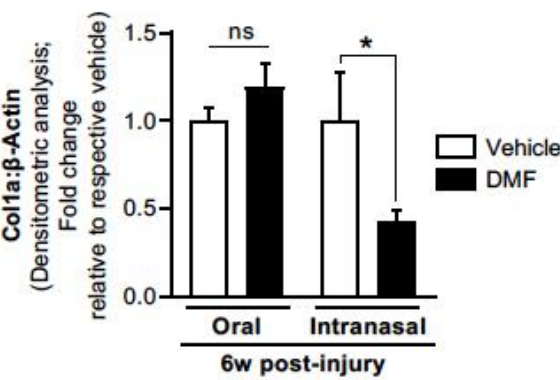

Figure 4. Intranasal (but not oral gavage) administration of DMF promotes reversal of age-associated established lung fibrosis. (A) Lung tissue was assessed by H\&E staining for histopathology (top panels) and Masson's trichrome blue staining for collagen (bottom panels). (B-E) Lung protein extract was assessed for fibronectin $(\mathbf{B}, \mathbf{C})$ and collagen-1 $\alpha(\mathbf{D}, \mathbf{E})$ expression by Western blot and densitometric analysis. Representative Western blot images are shown. Oral-vehicle $(n=6)$; oral-DMF $(n=5)$; intranasal-vehicle $(n=4)$; intranasal-DMF $(n=6)$. (F) The resolution of fibrosis was analyzed by quantitative hydroxyproline assay. Oral-vehicle $(n=5)$; oral-DMF $(n=5)$; intranasal-vehicle $(n=5)$; intranasal-DMF $(n=6)$. All values represent means of biological replicates \pm SEM; ns: not significant; ${ }^{*} p<0.05$ using Student's two-tailed $t$-test. 


\subsection{Intranasal DMF Delivery Promotes Resolution of Age-Dependent Established Fibrosis}

Inhaled drug delivery offers advantages over systemic (oral) drug administration for lung-targeted indications, as higher drug concentrations can be delivered directly to the lungs while potentially reducing the concentration of systemic exposure among other organs. Therefore, we sought to evaluate the efficacy of lung-targeted DMF treatment (via inhaled delivery) for reversing age-dependent established fibrosis (as opposed to systemic delivery, which failed to demonstrate efficacy). Using the same injury model and treatment protocol, aged mice were administered DMF or vehicle daily via intranasal delivery from 3-6 weeks post-injury (Figure 3). Inhaled DMF treatment resulted in resolution of age-dependent established lung fibrosis, as demonstrated by histopathology (Figure 4A; top panels), reduced collagen deposition (Figure $4 \mathrm{~A}$; bottom panels), significantly decreased expression levels of fibronectin (Figure $4 B, C$ ) and collagen- $1 \alpha$ (Figure $4 D, E$ ), and decreased lung hydroxyproline levels (Figure $4 \mathrm{~F}$ ) as compared to vehicle-treated and oral DMF treated mice (Figure 4A-F). In summary, these data suggest that, although systemic DMF delivery failed to reverse established fibrosis, lung-targeted DMF treatment via inhaled delivery demonstrated efficacy for promoting the resolution of age-dependent established lung fibrosis.

\subsection{Intranasal DMF Delivery Rescues Deficient Nrf2-Mediated Antioxidant Responses and Promotes Lung Redox Homeostasis}

Given that lung-targeted inhaled delivery of DMF demonstrated efficacy for promoting the resolution of age-dependent established lung fibrosis, whereas systemic oral delivery failed to demonstrate efficacy, we next evaluated the pharmacodynamics of oral vs. inhaled DMF delivery. Although oral administration of DMF led to reduced blood ROS levels (Figure 5A), it failed to rescue deficient Nrf2 expression levels in the lungs of aged mice with established fibrosis (Figure 5B,C). In contrast, although intranasal DMF delivery did not impact blood ROS levels (Figure 5A), it resulted in significantly increased lung Nrf2 expression levels (Figure 5B,C). Further, intranasal DMF delivery also led to significantly reduced oxidized glutathione levels (Figure 5D) and lipid peroxidation (Figure 5E) in the lungs, as compared to vehicle or oral DMF treatment. These data suggest that inhaled DMF delivery promotes Nrf2-mediated lung antioxidant responses to rescue lung redox imbalance without affecting systemic redox levels. In summary, lung-targeted treatment with DMF via inhaled delivery (as compared to oral delivery) can more effectively rescue local Nrf2 expression levels and promote redox homeostasis in the lungs.

A

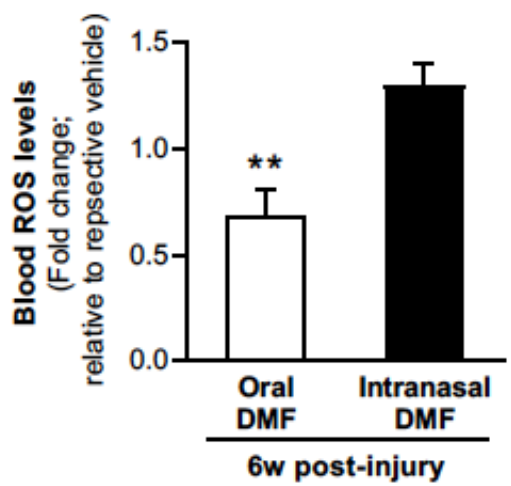

Figure 5. Cont. 
B

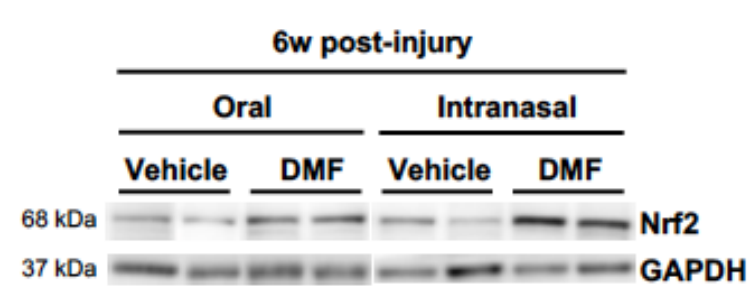

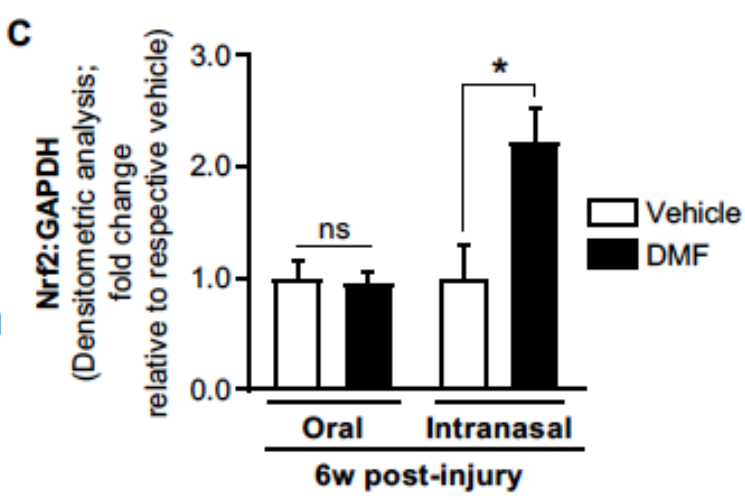

E

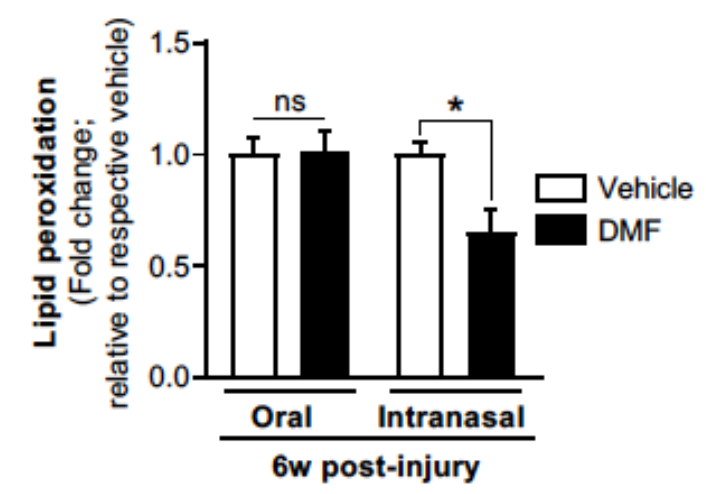

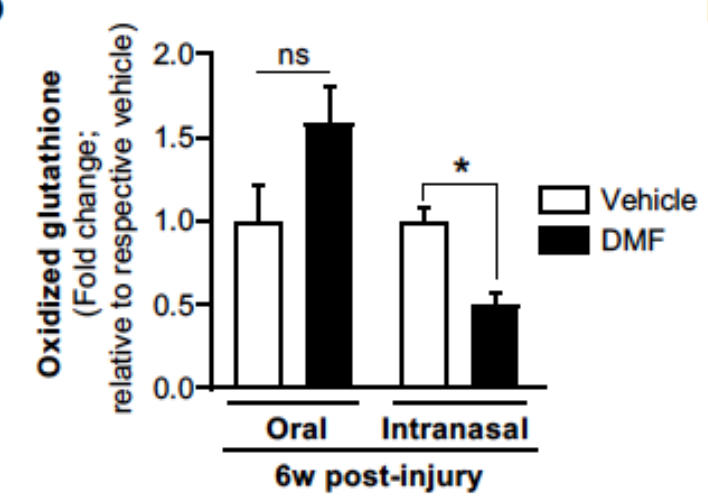

Figure 5. Intranasal (but not oral gavage) administration of DMF restores lung Nrf2 levels in aged-injured mice. (A) Blood ROS levels were analyzed by Amplex Red assay. Oral-DMF $(n=7)$; intranasal-DMF $(n=6)$. (B,C) Lung protein extract was assessed for Nrf2 expression by Western blot (B) and densitometric analysis (C). Representative Western blot images are shown. Oral-vehicle $(n=7)$; oral-DMF $(n=7)$; intranasal-vehicle $(n=4)$; intranasal-DMF $(n=4)$. (D) The level of lung oxidized glutathione was analyzed by a quantitative oxidized glutathione assay. Oral-vehicle $(n=5)$; oral-DMF $(n=5)$; intranasal-vehicle $(n=3)$; intranasal-DMF $(n=4)$. (E) The level of lung lipid peroxidation was analyzed by quantitative lipid peroxidation assay. Oral-vehicle $(n=5)$; oral-DMF $(n=5)$; intranasal-vehicle $(n=4)$; intranasal-DMF $(n=3)$. All values represent means of biological replicates \pm SEM; ns: not significant; ${ }^{*} p<0.05 ;{ }^{* *} p<0.01$ using Student's two-tailed $t$-test.

\section{Discussion}

Oxidative stress has long been associated with fibrotic disorders, including IPF [34]. Despite the clear link between aging and oxidative stress, therapeutically tenable agents that target age-associated oxidative stress to treat fibrosis have yet to be translated into a viable treatment for patients with IPF. Pre-clinical studies demonstrated a critical role for Nrf2 in mediating antioxidant responses during injury-repair. Further, Nrf2 activators, including sulforaphane [26] and DMF [25], demonstrated efficacy for inhibiting the development of fibrosis in young mice. However, in aged mice, lung injury results in persistent fibrosis, which is associated with defective Nrf2-mediated antioxidant responses [19]. Further, Nrf2 expression was reportedly decreased in the lungs of patients diagnosed with IPF [20]. Although therapeutic strategies targeting Nrf2 for the treatment of IPF hold promise, Nrf2targeted strategies yet to be evaluated in clinical trials for IPF. Systemic delivery of the Nrf2 activator, Bardoxolone methyl, was terminated in phase III clinical trials for pulmonary hypertension due to safety concerns, suggesting that long-term systemic treatment with some Nrf2 activators may not be appropriate for chronic diseases such as IPF. Tecfidera (an Nrf2 activator via the active pharmaceutical ingredient, DMF) is an FDA-approved drug for multiple sclerosis via oral administration. However, DMF was not evaluated for its 
efficacy in reversing age-associated established fibrosis. We hypothesized that strategies that more directly target the source(s) of redox imbalance via localized tissue-specific delivery would offer greater potential to reduce unintended side-effects and would be more effective as compared to systemic delivery for reversing age-associated established fibrosis. Our studies indicate that while systemic (oral) DMF delivery attenuated blood ROS levels, it failed to rescue lung Nrf2 levels and did not demonstrate efficacy for resolving age-dependent established lung fibrosis. Importantly, lung-targeted DMF delivery rescued deficient Nrf2-mediated antioxidant responses within the lung and promoted the resolution of age-dependent established lung fibrosis. The current study provides a side-by-side comparison of systemic vs. local delivery of DMF and demonstrates proof-of-concept for a lung-targeted antioxidant approach as a therapeutic strategy for reversing age-associated established fibrosis.

A major limitation of the traditional bleomycin-induced lung fibrosis model in young mice is the resolving nature of fibrosis, as lung injury results in a limited fibrotic response which resolves beyond 3 weeks post-injury $[19,33]$. One potential explanation for the limited clinical translation of therapeutics for IPF is that age-dependent pathologic mechanisms remain largely unexploited in the drug development process, despite the fact that aging is strongly implicated in the pathogenesis of IPF [34,35]. Pre-clinical animal models of lung fibrosis are largely performed in young mice (8-10 weeks), which predominantly results in a self-limited fibrotic response [36]. Treatment interventions are largely preventative (dosing before or at the time of injury) rather than curative [37]. Previous pre-clinical studies demonstrating the efficacy of Nrf2 activators for inhibiting the development of lung fibrosis in young animals using preventative treatment schedules $[25,26]$ are useful for initial proof-of-concept. However, it has become increasingly important to re-evaluate the use of this model for pre-clinical studies to predict therapeutic benefit in subsequent clinical trials, particularly since numerous drug candidates demonstrating efficacy in young mice have failed in clinical trials with elderly patients. This aging murine model of non-resolving lung fibrosis better recapitulates pathological signaling observed in human IPF patients (e.g., defective Nrf2 responsiveness), as limited knowledge of age-dependent pathological mechanisms / targets remain key challenges and may in part explain the limited therapies available. This is the first study that leverages an animal model of persistent lung fibrosis to evaluate the pre-clinical efficacy of an Nrf2 activator DMF for reversing established fibrosis (versus evaluating the pre-clinical efficacy of inhibiting de novo synthesis of fibrosis); this is a more clinically relevant efficacy testing protocol since patients with IPF typically present with well-established fibrosis. With the emergence of senolytics as a new potential strategy for IPF $[38,39]$, age-relevant pre-clinical efficacy models have become increasingly important to enhance the potential for clinical translation. Gender differences exist in response to bleomycin-induced lung injury [31]; therefore, we used female mice only to avoid sex-related variability of responses to injury. Future studies could further validate these findings in male mice. Overall, this study sheds light on the utility of aging animal models, as they provide opportunities to perform more rigorous efficacy testing that may enable the selection of more effective drug candidates, such as Tecfidera, which can promote the resolution of age-dependent established fibrosis. Leveraging aging models at the appropriate stages of therapeutic development is likely to provide novel insight that would accelerate the successful translation of improved therapies for IPF.

The two currently FDA-approved drugs for IPF slow disease progression, although they are not shown to reverse fibrosis [5], and neither drug is shown to definitively improve quality of life or survival for patients. Further, a number of side effects are reported for these drugs, which are administered via the oral route. There are currently two blockbuster dry powder inhalers (DPI) used most in the U.S. and globally, Advair and Spiriva (both are prescribed for asthma and COPD indications). From the clinical standpoint, lungtargeted drug delivery offers several major advantages over systemic delivery routes, including the ability to deliver higher local drug concentration and the potential for reduced side effects. Our study indicates that lung-targeted delivery of DMF, but not systemic 
delivery, mediates upregulation of Nrf2 expression, antioxidant activity, and promotes partial resolution of age-dependent established lung fibrosis. Of note, although the oral dose was three times higher $(12 \mathrm{mg} / \mathrm{kg})$ than the inhaled dose $(4 \mathrm{mg} / \mathrm{kg})$, only inhaled delivery demonstrated efficacy for promoting reversal of established fibrosis, despite the lower dose. Thus, although current IPF therapies are administered orally, lung-targeted delivery of novel therapeutics may provide greater efficacy (even at lower doses with reduced side effects. To date, no therapeutics have been shown to reverse age-associated established fibrosis, which may represent the holy grail for therapeutic strategies to more effectively treat IPF. A better understanding of injury-repair in the context of aging and/or efficacy evaluation of drug candidates in age-relevant pre-clinical models are likely to more accurately predict clinical translation of therapies for IPF, a disease that disproportionately afflicts the elderly population.

Supplementary Materials: The following supporting information can be downloaded at: https: / /www.mdpi.com/article/10.3390/antiox11030492/s1.

Author Contributions: L.H. conceived of the project, provided funding, and supervised all studies. L.H., K.K., I.P., Y.-J.S., J.M.K., S.P., J.D.I., S.P.R., K.S.K. and S.H. designed, conducted, and / or performed analyses for experiments. All authors contributed intellectual input to the overall project, technical support, analyses of data, and/or manuscript preparation. All authors have read and agreed to the published version of the manuscript.

Funding: This work was supported by the National Institutes of Health grant 1R21AG054766-01 (LH) and the Veterans Administration Health System grant 1 I01 BX003919-01A1 (LH).

Institutional Review Board Statement: The study was conducted according to the guidelines of the Declaration of Helsinki and approved by the Institutional Review Board of the University of Arizona (\#1200000347).

Informed Consent Statement: Informed consent was obtained from all participants involved in the study.

Data Availability Statement: Data is contained within the article.

Conflicts of Interest: The authors declare no conflict of interest.

\section{References}

1. Duffield, J.S.; Lupher, M.; Thannickal, V.J.; Wynn, T.A. Host responses in tissue repair and fibrosis. Annu. Rev. Pathol. 2013, 8, 241-276. [CrossRef]

2. Wynn, T.A. Fibrotic disease and the $\mathrm{T}(\mathrm{H}) 1 / \mathrm{T}(\mathrm{H}) 2$ paradigm. Nat. Rev. Immunol. 2004, 4, 583-594. [CrossRef] [PubMed]

3. Raghu, G.; Collard, H.R.; Egan, J.J.; Martinez, F.J.; Behr, J.; Brown, K.K.; Colby, T.V.; Cordier, J.F.; Flaherty, K.R.; Lasky, J.A.; et al. An official ATS/ERS/JRS/ALAT statement: Idiopathic pulmonary fibrosis: Evidence-based guidelines for diagnosis and management. Am. J. Respir. Crit. Care Med. 2011, 183, 788-824. [CrossRef] [PubMed]

4. Raghu, G.; Weycker, D.; Edelsberg, J.; Bradford, W.Z.; Oster, G. Incidence and prevalence of idiopathic pulmonary fibrosis. Am. J. Respir. Crit. Care Med. 2006, 174, 810-816. [CrossRef] [PubMed]

5. Kato, K.; Shin, Y.J.; Palumbo, S.; Papageorgiou, I.; Hahn, S.; Irish, J.D.; Rounseville, S.P.; Krafty, R.T.; Wollin, L.; Sauler, M.; et al. Leveraging aging models of pulmonary fibrosis; the efficacy of nintedanib in aging. Eur. Respir. J. 2021, 58, 2100759. [CrossRef] [PubMed]

6. Gagliano, N.; Grizzi, F.; Annoni, G. Mechanisms of aging and liver functions. Dig. Dis. 2007, 25, 118-123. [CrossRef]

7. Gagliano, N.; Arosio, B.; Santambrogio, D.; Balestrieri, M.R.; Padoani, G.; Tagliabue, J.; Masson, S.; Vergani, C.; Annoni, G. Age-dependent expression of fibrosis-related genes and collagen deposition in rat kidney cortex. J. Gerontol. A Biol. Sci. Med. Sci. 2000, 55, B365-B372. [CrossRef] [PubMed]

8. Glaser, J.; Stienecker, K. Pancreas and aging: A study using ultrasonography. Gerontology 2000, 46, 93-96. [CrossRef]

9. Calabresi, C.; Arosio, B.; Galimberti, L.; Scanziani, E.; Bergottini, R.; Annoni, G.; Vergani, C. Natural aging, expression of fibrosis-related genes and collagen deposition in rat lung. Exp. Gerontol. 2007, 42, 1003-1011. [CrossRef]

10. Fell, C.D.; Martinez, F.J.; Liu, L.X.; Murray, S.; Han, M.K.; Kazerooni, E.A.; Gross, B.H.; Myers, J.; Travis, W.D.; Colby, T.V.; et al. Clinical predictors of a diagnosis of idiopathic pulmonary fibrosis. Am. J. Respir. Crit. Care Med. 2010, 181, 832-837. [CrossRef]

11. Hecker, L.; Thannickal, V.J. Nonresolving fibrotic disorders: Idiopathic pulmonary fibrosis as a paradigm of impaired tissue regeneration. Am. J. Med. Sci. 2011, 341, 431-434. [CrossRef] [PubMed]

12. Collard, H.R. The age of idiopathic pulmonary fibrosis. Am. J. Respir. Crit. Care Med. 2010, 181, 771-772. [CrossRef] [PubMed] 
13. Cantin, A.M.; Hubbard, R.C.; Crystal, R.G. Glutathione deficiency in the epithelial lining fluid of the lower respiratory tract in idiopathic pulmonary fibrosis. Am. Rev. Respir. Dis. 1989, 139, 370-372. [CrossRef]

14. Kinnula, V.L.; Hodgson, U.A.; Lakari, E.K.; Tan, R.J.; Sormunen, R.T.; Soini, Y.M.; Kakko, S.J.; Laitinen, T.H.; Oury, T.D.; Paakko, P.K. Extracellular superoxide dismutase has a highly specific localization in idiopathic pulmonary fibrosis/usual interstitial pneumonia. Histopathology 2006, 49, 66-74. [CrossRef] [PubMed]

15. Beeh, K.M.; Beier, J.; Haas, I.C.; Kornmann, O.; Micke, P.; Buhl, R. Glutathione deficiency of the lower respiratory tract in patients with idiopathic pulmonary fibrosis. Eur. Respir. J. 2002, 19, 1119-1123. [CrossRef] [PubMed]

16. Psathakis, K.; Mermigkis, D.; Papatheodorou, G.; Loukides, S.; Panagou, P.; Polychronopoulos, V.; Siafakas, N.M.; Bouros, D. Exhaled markers of oxidative stress in idiopathic pulmonary fibrosis. Eur. J. Clin. Investig. 2006, 36, 362-367. [CrossRef]

17. Lenz, A.G.; Costabel, U.; Maier, K.L. Oxidized BAL fluid proteins in patients with interstitial lung diseases. Eur. Respir. J. 1996, 9 , 307-312. [CrossRef]

18. Maier, K.; Leuschel, L.; Costabel, U. Increased levels of oxidized methionine residues in bronchoalveolar lavage fluid proteins from patients with idiopathic pulmonary fibrosis. Am. Rev. Respir. Dis. 1991, 143, 271-274. [CrossRef]

19. Hecker, L.; Logsdon, N.J.; Kurundkar, D.; Kurundkar, A.; Bernard, K.; Hock, T.; Meldrum, E.; Sanders, Y.Y.; Thannickal, V.J. Reversal of persistent fibrosis in aging by targeting Nox4-Nrf2 redox imbalance. Sci. Transl. Med. 2014, 6, 231ra247. [CrossRef]

20. Artaud-Macari, E.; Goven, D.; Brayer, S.; Hamimi, A.; Besnard, V.; Marchal-Somme, J.; Ali, Z.E.; Crestani, B.; Kerdine-Romer, S.; Boutten, A.; et al. Nuclear factor erythroid 2-related factor 2 nuclear translocation induces myofibroblastic dedifferentiation in idiopathic pulmonary fibrosis. Antioxid. Redox Signal. 2013, 18, 66-79. [CrossRef]

21. Kinnula, V.L.; Fattman, C.L.; Tan, R.J.; Oury, T.D. Oxidative stress in pulmonary fibrosis: A possible role for redox modulatory therapy. Am. J. Respir. Crit. Care Med. 2005, 172, 417-422. [CrossRef] [PubMed]

22. Zhou, W.; Mo, X.; Cui, W.; Zhang, Z.; Li, D.; Li, L.; Xu, L.; Yao, H.; Gao, J. Nrf2 inhibits epithelial-mesenchymal transition by suppressing snail expression during pulmonary fibrosis. Sci. Rep. 2016, 6, 38646. [CrossRef] [PubMed]

23. Kikuchi, N.; Ishii, Y.; Morishima, Y.; Yageta, Y.; Haraguchi, N.; Itoh, K.; Yamamoto, M.; Hizawa, N. Nrf2 protects against pulmonary fibrosis by regulating the lung oxidant level and Th1/Th2 balance. Respir. Res. 2010, 11, 31. [CrossRef] [PubMed]

24. Cho, H.Y.; Reddy, S.P.; Yamamoto, M.; Kleeberger, S.R. The transcription factor NRF2 protects against pulmonary fibrosis. FASEB J. 2004, 18, 1258-1260. [CrossRef] [PubMed]

25. Grzegorzewska, A.P.; Seta, F.; Han, R.; Czajka, C.A.; Makino, K.; Stawski, L.; Isenberg, J.S.; Browning, J.L.; Trojanowska, M Dimethyl Fumarate ameliorates pulmonary arterial hypertension and lung fibrosis by targeting multiple pathways. Sci. Rep. 2017, 7, 41605. [CrossRef] [PubMed]

26. Yan, B.; Ma, Z.; Shi, S.; Hu, Y.; Ma, T.; Rong, G.; Yang, J. Sulforaphane prevents bleomycininduced pulmonary fibrosis in mice by inhibiting oxidative stress via nuclear factor erythroid 2related factor2 activation. Mol. Med. Rep. 2017, 15, 4005-4014. [CrossRef]

27. Idiopathic Pulmonary Fibrosis Clinical Research, N.; Martinez, F.J.; de Andrade, J.A.; Anstrom, K.J.; King, T.E., Jr.; Raghu, G. Randomized trial of acetylcysteine in idiopathic pulmonary fibrosis. N. Engl. J. Med. 2014, 370, 2093-2101. [CrossRef]

28. Linker, R.A.; Lee, D.H.; Ryan, S.; van Dam, A.M.; Conrad, R.; Bista, P.; Zeng, W.; Hronowsky, X.; Buko, A.; Chollate, S.; et al. Fumaric acid esters exert neuroprotective effects in neuroinflammation via activation of the Nrf2 antioxidant pathway. Brain 2011, 134, 678-692. [CrossRef]

29. Palumbo, S.; Shin, Y.J.; Ahmad, K.; Desai, A.A.; Quijada, H.; Mohamed, M.; Knox, A.; Sammani, S.; Colson, B.A.; Wang, T.; et al. Dysregulated Nox4 ubiquitination contributes to redox imbalance and age-related severity of acute lung injury. Am. J. Physiol. Lung Cell. Mol. Physiol. 2017, 312, L297-L308. [CrossRef]

30. Kato, K.; Logsdon, N.J.; Shin, Y.J.; Palumbo, S.; Knox, A.; Irish, J.D.; Rounseville, S.P.; Rummel, S.R.; Mohamed, M.; Ahmad, K.; et al. Impaired Myofibroblast Dedifferentiation Contributes to Non-Resolving Fibrosis in Aging. Am. J. Respir. Cell Mol. Biol. 2020, 62, 633-644. [CrossRef]

31. Redente, E.F.; Jacobsen, K.M.; Solomon, J.J.; Lara, A.R.; Faubel, S.; Keith, R.C.; Henson, P.M.; Downey, G.P.; Riches, D.W. Age and sex dimorphisms contribute to the severity of bleomycin-induced lung injury and fibrosis. Am. J. Physiol. Lung Cell Mol. Physiol. 2011, 301, L510-L518. [CrossRef] [PubMed]

32. Hecker, L.; Vittal, R.; Jones, T.; Jagirdar, R.; Luckhardt, T.R.; Horowitz, J.C.; Pennathur, S.; Martinez, F.J.; Thannickal, V.J. NADPH oxidase-4 mediates myofibroblast activation and fibrogenic responses to lung injury. Nat. Med. 2009, 15, 1077-1081. [CrossRef] [PubMed]

33. Izbicki, G.; Segel, M.J.; Christensen, T.G.; Conner, M.W.; Breuer, R. Time course of bleomycin-induced lung fibrosis. Int. J. Exp. Pathol. 2002, 83, 111-119. [CrossRef] [PubMed]

34. Kato, K.; Hecker, L. NADPH oxidases: Pathophysiology and therapeutic potential in age-associated pulmonary fibrosis. Redox Biol. 2020, 33, 101541. [CrossRef] [PubMed]

35. Hecker, L. Mechanisms and consequences of oxidative stress in lung disease: Therapeutic implications for an aging populace. Am. J. Physiol. Lung Cell. Mol. Physiol. 2018, 314, L642-L653. [CrossRef] [PubMed]

36. Moore, B.B.; Hogaboam, C.M. Murine models of pulmonary fibrosis. Am. J. Physiology. Lung Cell. Mol. Physiol. 2008, 294, L152-L160. [CrossRef] [PubMed]

37. Moeller, A.; Ask, K.; Warburton, D.; Gauldie, J.; Kolb, M. The bleomycin animal model: A useful tool to investigate treatment options for idiopathic pulmonary fibrosis? Int. J. Biochem. Cell Biol. 2008, 40, 362-382. [CrossRef] 
38. Brandsma, C.A.; de Vries, M.; Costa, R.; Woldhuis, R.R.; Konigshoff, M.; Timens, W. Lung ageing and COPD: Is there a role for ageing in abnormal tissue repair? Eur. Respir. Rev. 2017, 26, 170073. [CrossRef]

39. Merkt, W.; Bueno, M.; Mora, A.L.; Lagares, D. Senotherapeutics: Targeting senescence in idiopathic pulmonary fibrosis. Semin. Cell Dev. Biol. 2020, 101, 104-110. [CrossRef] 